\title{
Search for the decay $\eta^{\prime} \rightarrow \gamma \gamma \eta$
}

M. Ablikim, ${ }^{1}$ M. N. Achasov,${ }^{10, d}$ P. Adlarson,${ }^{59}$ S. Ahmed,${ }^{15}$ M. Albrecht,${ }^{4}$ M. Alekseev ${ }^{58 a, 58 c}$ A. Amoroso, ${ }^{58 a, 58 c}$ F. F. An, ${ }^{1}$ Q. An ${ }^{55,43}$ Y. Bai, ${ }^{42}$ O. Bakina, ${ }^{27}$ R. Baldini Ferroli,${ }^{23 a}$ I. Balossino, ${ }^{24 a}$ Y. Ban, ${ }^{35}$ K. Begzsuren,${ }^{25}$ J. V. Bennett, ${ }^{5}$ N. Berger ${ }^{26}$ M. Bertani, ${ }^{23 a}$ D. Bettoni, ${ }^{24 a}$ F. Bianchi,${ }^{58,58 c}$ J. Biernat, ${ }^{59}$ J. Bloms ${ }^{52}$ I. Boyko, ${ }^{27}$ R. A. Briere, ${ }^{5}$ H. Cai, ${ }^{60}$ X. Cai, ${ }^{1,43}$ A. Calcaterra, ${ }^{23 a}$ G. F. Cao ${ }^{1,47}$ N. Cao, ${ }^{1,47}$ S. A. Cetin, ${ }^{46 \mathrm{~b}}$ J. Chai, ${ }^{58 \mathrm{c}}$ J. F. Chang, ${ }^{1,43}$ W. L. Chang ${ }_{1,47}^{1,47}$ G. Chelkov, ${ }^{27, b, c}$ D. Y. Chen, ${ }^{6}$ G. Chen, ${ }^{1}$ H. S. Chen, ${ }^{1,47}$ J. C. Chen, ${ }^{1}$ M. L. Chen, ${ }^{1,43}$ S. J. Chen, ${ }^{33}$ Y. B. Chen, ${ }^{1,43}$ W. Cheng, ${ }^{58 c}$ G. Cibinetto ${ }^{24 a}$ F. Cossio, ${ }^{58 c}$ X. F. Cui,${ }^{34}$ H. L. Dai ${ }^{1,43}$ J. P. Dai ${ }^{38, h}$ X. C. Dai,${ }^{1,47}$ A. Dbeyssi,${ }^{15}$ D. Dedovich,${ }^{27}$ Z. Y. Deng, ${ }^{1}$ A. Denig, ${ }^{26}$ I. Denysenko, ${ }^{27}$ M. Destefanis, ${ }^{58 a, 58 c}$ F. De Mori ${ }^{58 a, 58 c}$ Y. Ding,${ }^{31}$ C. Dong, ${ }^{34}$ J. Dong, ${ }^{1,43}$ L. Y. Dong,,${ }^{1,47}$ M. Y. Dong, ${ }^{1,43,47}$ Z. L. Dou, ${ }^{33}$ S. X. Du, ${ }^{63}$ J. Z. Fan, ${ }^{45}$ J. Fang,,${ }^{1,43}$ S. S. Fang, ${ }^{1,47}$ Y. Fang, ${ }^{1}$ R. Farinelli, ${ }^{24 a, 24 b}$ L. Fava, ${ }^{58 b, 58 c}$ F. Feldbauer, ${ }^{4}$ G. Felici, ${ }^{23 a}$ C. Q. Feng, ${ }^{55,43}$ M. Fritsch, ${ }^{4}$ C. D. Fu, ${ }^{1}$ Y. Fu, ${ }^{1}$ Q. Gao, ${ }^{1}$ X. L. Gao, ${ }^{55,43}$ Y. Gao,${ }^{56}$ Y. Gao, ${ }^{45}$

Y. G. Gao, ${ }^{6}$ Z. Gao ${ }^{55,43}$ B. Garillon, ${ }^{26}$ I. Garzia, ${ }^{24 a}$ E. M. Gersabeck, ${ }^{50}$ A. Gilman, ${ }^{51}$ K. Goetzen, ${ }^{11}$ L. Gong, ${ }^{34}$ W. X. Gong, ${ }^{1,43}$ W. Gradl, ${ }^{26}$ M. Greco ${ }^{58 a, 58 c}$ L. M. Gu, ${ }^{33}$ M. H. Gu, ${ }^{1,43}$ S. Gu, ${ }^{2}$ Y. T. Gu, ${ }^{13}$ A. Q. Guo, ${ }^{22}$ L. B. Guo, ${ }^{32}$ R. P. Guo, ${ }^{36}$ Y. P. Guo, ${ }^{26}$ A. Guskov, ${ }^{27}$ S. Han, ${ }^{60}$ X. Q. Hao,${ }^{16}$ F. A. Harris, ${ }^{48}$ K. L. He, ${ }^{1,47}$ F. H. Heinsius, ${ }^{4}$ T. Held, ${ }^{4}$

Y. K. Heng ${ }^{1,43,47}$ M. Himmelreich, ${ }^{11, g}$ Y. R. Hou, ${ }^{47}$ Z. L. Hou, ${ }^{1}$ H. M. Hu ${ }^{1,47}$ J. F. Hu, ${ }^{38, h}$ T. Hu, ${ }^{1,43,47}$ Y. Hu, ${ }^{1}$ G. S. Huang, ${ }^{5,43}$ J. S. Huang, ${ }^{16}$ X. T. Huang, ${ }^{37}$ X. Z. Huang, ${ }^{33}$ N. Huesken, ${ }^{52}$ T. Hussain, ${ }^{57}$ W. Ikegami Andersson, ${ }^{59}$ W. Imoehl ${ }^{22}$ M. Irshad ${ }^{55,43}$ Q. Ji, ${ }^{1}$ Q. P. Ji, ${ }^{16}$ X. B. Ji, ${ }^{1,47}$ X. L. Ji, ${ }^{1,43}$ H. L. Jiang, ${ }^{37}$ X. S. Jiang, ${ }^{1,43,47}$ X. Y. Jiang, ${ }^{34}$

J. B. Jiao, ${ }^{37}$ Z. Jiao ${ }^{18}$ D. P. Jin, ${ }^{1,43,47}$ S. Jin ${ }^{33}$ Y. Jin, ${ }^{49}$ T. Johansson, ${ }^{59}$ N. Kalantar-Nayestanaki, ${ }^{29}$ X. S. Kang, ${ }^{31}$ R. Kappert, ${ }^{29}$ M. Kavatsyuk, ${ }^{29}$ B. C. Ke, ${ }^{1}$ I. K. Keshk, ${ }^{4}$ A. Khoukaz, ${ }^{52}$ P. Kiese, ${ }^{26}$ R. Kiuchi, ${ }^{1}$ R. Kliemt,${ }^{11}$ L. Koch, ${ }^{28}$ O. B. Kolcu, ${ }^{46 b, f}$ B. Kopf, ${ }^{4}$ M. Kuemmel, ${ }^{4}$ M. Kuessner, ${ }^{4}$ A. Kupsc, ${ }^{59}$ M. Kurth, ${ }^{1}$ M. G. Kurth,${ }^{1,47}$ W. Kühn, ${ }^{28}$ J. S. Lange, ${ }^{28}$ P. Larin, ${ }^{15}$ L. Lavezzi, ${ }^{58 c}$ H. Leithoff, ${ }^{26}$ T. Lenz,${ }^{26}$ C. Li ${ }^{59}$ Cheng Li,${ }^{55,43}$ D. M. Li, ${ }^{63} \mathrm{~F}$. Li, ${ }^{1,43}{ }^{5}$ F. Y. Li, ${ }^{35}$ G. Li ${ }^{1}{ }^{1}$ H. B. Li,${ }^{1,47}$ H. J. Li, ${ }^{9, j}$ J. C. Li, ${ }^{1}$ J. W. Li, ${ }^{41} \mathrm{Ke} \mathrm{Li},{ }^{1}$ L. K. Li, ${ }^{1}$ Lei Li, ${ }^{3}$ P. L. Li, ${ }^{55,43}$ P. R. Li, ${ }^{30}$ Q. Y. Li, ${ }^{37}$ W. D. Li, ${ }^{1,47}$ W. G. Li, ${ }^{1}$ X. H. Li ${ }^{55,43}$ X. L. Li,${ }^{37}$ X. N. Li ${ }^{1,43}$ Z. B. Li, ${ }^{44}$ Z. Y. Li ${ }^{44}$ H. Liang, ${ }^{55,43}$ H. Liang, ${ }^{1,47}$ Y. F. Liang, ${ }^{40}$ Y. T. Liang, ${ }^{28}$

G. R. Liao, ${ }^{12}$ L. Z. Liao ${ }^{1,47}$ J. Libby, ${ }^{21}$ C. X. Lin, ${ }^{44}$ D. X. Lin ${ }^{15}$ Y. J. Lin, ${ }^{13}$ B. Liu, ${ }^{38, h}$ B. J. Liu, ${ }^{1}$ C. X. Liu, ${ }^{1}$ D. Liu, $, 5,43$

D. Y. Liu, ${ }^{38, h}$ F. H. Liu, ${ }^{39}$ Fang Liu, ${ }^{1}$ Feng Liu, ${ }^{6}$ H. B. Liu, ${ }^{13}$ H. M. Liu ${ }^{1,47}$ Huanhuan Liu, ${ }^{1}$ Huihui Liu, ${ }^{17}$ J. B. Liu, ${ }^{55,43}$ J. Y. Liu, ${ }^{1,47}$ K. Y. Liu, ${ }^{31}$ Ke Liu, ${ }^{6}$ L. Y. Liu, ${ }^{13}$ Q. Liu, ${ }^{47}$ S. B. Liu, ${ }^{55,43}$ T. Liu, ${ }^{1,47}$ X. Liu, ${ }^{30}$ X. Y. Liu,,${ }^{1,47}$ Y. B. Liu, ${ }^{34}$ Z. A. Liu, ${ }^{1,43,47}$ Zhiqing Liu, ${ }^{37}$ Y. F. Long, ${ }^{35}$ X. C. Lou, ${ }^{1,43,47}$ H. J. Lu, ${ }^{18}$ J. D. Lu, ${ }^{1,47}$ J. G. Lu, ${ }^{1,43}$ Y. Lu, ${ }^{1}$ Y. P. Lu, ${ }^{1,43}$ C. L. Luo, ${ }^{32}$ M. X. Luo, ${ }^{62}$ P. W. Luo, ${ }^{44}$ T. Luo, ${ }^{9, j}$ X. L. Luo, ${ }^{1,43}$ S. Lusso, ${ }^{58 c}$ X. R. Lyu, ${ }^{47}$ F. C. Ma, ${ }^{31}$ H. L. Ma, ${ }^{1}$ L. L. Ma, ${ }^{37}$ M. M. Ma,${ }^{1,47}$ Q. M. Ma,${ }^{1}$ X. N. Ma,${ }^{34}$ X. X. Ma,${ }^{1,47}$ X. Y. Ma, ${ }^{1,43}$ Y. M. Ma ${ }^{37}$ F. E. Maas, ${ }^{15}$ M. Maggiora, ${ }^{58,58 c}$

S. Maldaner, ${ }^{26}$ S. Malde, ${ }^{53}$ Q. A. Malik,${ }^{57}$ A. Mangoni ${ }^{23 b}$ Y. J. Mao, ${ }^{35}$ Z. P. Mao, ${ }^{1}$ S. Marcello, ${ }^{58 a, 58 c}$ Z. X. Meng, ${ }^{49}$ J. G. Messchendorp, ${ }^{29}$ G. Mezzadri, ${ }^{24 a}$ J. Min,${ }^{1,43}$ T. J. Min, ${ }^{33}$ R. E. Mitchell, ${ }^{22}$ X. H. Mo, ${ }^{1,43,47}$ Y. J. Mo, ${ }^{6}$

C. Morales Morales, ${ }^{15}$ N. Yu. Muchnoi,${ }^{10, d}$ H. Muramatsu, ${ }^{51}$ A. Mustafa, ${ }^{4}$ S. Nakhoul, ${ }^{11, g}$ Y. Nefedov,${ }^{27}$ F. Nerling, ${ }^{11, g}$ I. B. Nikolaev, ${ }^{10, d}$ Z. Ning, ${ }^{1,43}$ S. Nisar, ${ }^{8, k}$ S. L. Niu, ${ }^{1,43}$ S. L. Olsen, ${ }^{47}$ Q. Ouyang, ${ }^{1,43,47}$ S. Pacetti, ${ }^{23 \mathrm{~b}}$ Y. Pan, ${ }^{55,43}$ M. Papenbrock, ${ }^{59}$ P. Patteri, ${ }^{23 a}$ M. Pelizaeus, ${ }^{4}$ H. P. Peng,${ }^{55,43}$ K. Peters, ${ }^{11,9}$ J. Pettersson, ${ }^{59}$ J. L. Ping, ${ }^{32}$ R. G. Ping, ${ }^{1,47}$ A. Pitka, ${ }^{4}$ R. Poling, ${ }^{51}$ V. Prasad,${ }^{55,43}$ H. R. Qi, ${ }^{2}$ M. Qi ${ }^{33}$ T. Y. Qi ${ }^{2}$ S. Qian, ${ }^{1,43}$ C. F. Qiao, ${ }^{47}$ N. Qin, ${ }^{60}$ X. P. Qin, ${ }^{13}$ X. S. Qin, ${ }^{4}$ Z. H. Qin, ${ }^{1,43}$ J. F. Qiu, ${ }^{1}$ S. Q. Qu, ${ }^{34}$ K. H. Rashid, ${ }^{57, i}$ K. Ravindran, ${ }^{21}$ C. F. Redmer, ${ }^{26}$ M. Richter, ${ }^{4}$ A. Rivetti, ${ }^{58 c}$ V. Rodin ${ }^{29}$ M. Rolo, ${ }^{58 \mathrm{c}}$ G. Rong, ${ }^{1,47}$ Ch. Rosner, ${ }^{15}$ M. Rump, ${ }^{52}$ A. Sarantsev, ${ }^{27, \mathrm{e}}$ M. Savrié, ${ }^{24 \mathrm{~b}}$ Y. Schelhaas, ${ }^{26}$ K. Schoenning, ${ }^{59}$ W. Shan, ${ }^{19}$ X. Y. Shan, ${ }^{55,43}$ M. Shao, ${ }^{55,43}$ C. P. Shen, ${ }^{2}$ P. X. Shen, ${ }^{34}$ X. Y. Shen, ${ }^{1,47}$ H. Y. Sheng, ${ }^{1}$ X. Shi, ${ }^{1,43}$ X. D. Shi, ${ }^{55,43}$ J. J. Song, ${ }^{37}$ Q. Q. Song,${ }^{55,43}$ X. Y. Song, ${ }^{1}$ S. Sosio,${ }^{58 a, 58 c}$ C. Sowa ${ }^{4}$ S. Spataro, ${ }^{58 a, 58 c}$ F. F. Sui,${ }^{37}$ G. X. Sun, ${ }^{1}$ J. F. Sun ${ }^{16}$ L. Sun, ${ }^{60}$ S. S. Sun, ${ }^{1,47}$ X. H. Sun, ${ }^{1}$ Y. J. Sun, ${ }^{55,43}$ Y. K. Sun, ${ }^{55,43}$ Y. Z. Sun, ${ }^{1}$ Z. J. Sun ${ }^{1,43}$ Z. T. Sun, ${ }^{1}$ Y. T. Tan, ${ }^{55,43}$ C. J. Tang, ${ }^{40}$ G. Y. Tang, ${ }^{1}$ X. Tang, ${ }^{1}$ V. Thoren, ${ }^{59}$ B. Tsednee, ${ }^{25}$ I. Uman, ${ }^{46 d}$ B. Wang, ${ }^{1}$ B. L. Wang, ${ }_{47}$ C. W. Wang, ${ }^{33}$

D. Y. Wang, ${ }^{35}$ K. Wang, ${ }^{1,43}$ L. L. Wang, ${ }^{1}$ L. S. Wang, ${ }^{1}$ M. Wang, ${ }^{37}$ M. Z. Wang, ${ }^{35}$ Meng Wang, ${ }^{1,47}$ P. L. Wang, ${ }^{1}$

R. M. Wang, ${ }^{61}$ W. P. Wang, ${ }^{55,43}$ X. Wang, ${ }^{35}$ X. F. Wang, ${ }^{1}$ X. L. Wang, ${ }^{9, j}$ Y. Wang, ${ }^{44}$ Y. Wang, ${ }^{55,43}$ Y. F. Wang, ${ }^{1,43,47}$ Y. Q. Wang $\odot,{ }^{1,47}$ Z. Wang, ${ }^{1,43}$ Z. G. Wang, ${ }^{1,43}$ Z. Y. Wang, ${ }^{1}$ Zongyuan Wang, ${ }^{1,47}$ T. Weber, ${ }^{4}$ D. H. Wei, ${ }^{12}$ P. Weidenkaff, ${ }^{26}$ H. W. Wen ${ }^{32}$ S. P. Wen, ${ }^{1}$ U. Wiedner, ${ }^{4}$ G. Wilkinson, ${ }^{53}$ M. Wolke, ${ }^{59}$ L. H. Wu, ${ }^{1}$ L. J. Wu ${ }^{1,47}$ Z. Wu ${ }^{1,43}$ L. Xia, ${ }^{55,43}$ Y. Xia, ${ }^{20}$ S. Y. Xiao, ${ }^{1}$ Y. J. Xiao, ${ }^{1,47}$ Z. J. Xiao, ${ }^{32}$ Y. G. Xie, ${ }^{1,43}$ Y. H. Xie, ${ }^{6}$ T. Y. Xing, ${ }^{1,47}$ X. A. Xiong, ${ }^{1,47}$ Q. L. Xiu, ${ }^{1,43}$ G. F. Xu ${ }^{1}$ J. J. Xu, ${ }^{33}$ L. Xu, ${ }^{1}$ Q. J. Xu, ${ }^{14}$ W. Xu, ${ }^{1,47}$ X. P. Xu, ${ }^{41}$ F. Yan, ${ }^{56}$ L. Yan, ${ }^{58,58 c}$ W. B. Yan, ${ }^{55,43}$ W. C. Yan, ${ }^{2}$ Y. H. Yan, ${ }^{20}$ H. J. Yang, ${ }^{38, h}$ H. X. Yang, ${ }^{1}$ L. Yang, ${ }^{60}$ R. X. Yang, ${ }^{55,43}$ S. L. Yang, ${ }^{1,47}$ Y. H. Yang, ${ }^{33}$ Y. X. Yang, ${ }^{12}$ Yifan Yang, ${ }^{1,47}$ Z. Q. Yang ${ }^{20}{ }^{2}$ M. Ye, ${ }^{1,43}$ M. H. Ye, ${ }^{7}$ J. H. Yin, ${ }^{1}$ Z. Y. You, ${ }^{44}$ B. X. Yu, ${ }^{1,43,47}$ C. X. Yu, ${ }^{34}$ J. S. Yu, ${ }^{20}$ T. Yu, ${ }^{56}$ C. Z. Yuan, ${ }^{1,47}$ X. Q. Yuan, ${ }^{35}$ Y. Yuan, ${ }^{1}$ A. Yuncu, ${ }^{46 b, a}$ A. A. Zafar ${ }^{57}$ Y. Zeng, ${ }^{20}$ B. X. Zhang, ${ }^{1}$ B. Y. Zhang, ${ }_{1,43}$ C. C. Zhang, ${ }^{1}$ D. H. Zhang, ${ }^{1}$ H. H. Zhang, ${ }^{44}$ H. Y. Zhang, ${ }^{1,43}$ J. Zhang, ${ }^{1,47}$ J. L. Zhang, ${ }^{61}$ J. Q. Zhang, ${ }^{4}$ J. W. Zhang, ${ }^{1,43,47}$ J. Y. Zhang, ${ }^{1}$ J. Z. Zhang, ${ }^{1,47}$ K. Zhang, ${ }^{1,4}$ L. Zhang, ${ }^{45}$ S. F. Zhang, ${ }^{33}$ T. J. Zhang, ${ }^{38, h}$ X. Y. Zhang, ${ }^{37}$ Y. Zhang, ${ }^{5,, 43}$ Y. H. Zhang, ${ }^{1,43}$ Y. T. Zhang, ${ }^{55,43}$ Yang Zhang, ${ }^{1}$ Yao Zhang, ${ }^{1}$ Yi Zhang, ${ }^{, j}$ Yu Zhang, ${ }^{47}$ Z. H. Zhang, ${ }^{6}$ Z. P. Zhang, ${ }^{55}$ Z. Y. Zhang, ${ }^{60}$ G. Zhao, ${ }^{1}$ J. W. Zhao, ${ }^{1,43}$ 
J. Y. Zhao, ${ }^{1,47}$ J. Z. Zhao, ${ }^{1,43}$ Lei Zhao, ${ }^{55,43}$ Ling Zhao, ${ }^{1}$ M. G. Zhao, ${ }^{34}$ Q. Zhao, ${ }^{1}$ S. J. Zhao, ${ }^{63}$ T. C. Zhao, ${ }^{1}$ Y. B. Zhao, ${ }^{1,43}$ Z. G. Zhao, ${ }^{55,43}$ A. Zhemchugov, ${ }^{27, b}$ B. Zheng, ${ }^{56}$ J. P. Zheng, ${ }^{1,43}$ Y. Zheng, ${ }^{35}$ Y. H. Zheng, ${ }^{47}$ B. Zhong, ${ }^{32}$ L. Zhou, ${ }^{1,43}$ L. P. Zhou, ${ }^{1,47}$ Q. Zhou, ${ }^{1,47}$ X. Zhou, ${ }^{60}$ X. K. Zhou, ${ }^{47}$ X. R. Zhou, ${ }^{55,43}$ Xiaoyu Zhou, ${ }^{20}$ Xu Zhou, ${ }^{20}$ A. N. Zhu, ${ }^{1,47}$ J. Zhu, ${ }^{34}$ J. Zhu, ${ }^{44}$ K. Zhu, ${ }^{1}$ K. J. Zhu, ${ }^{1,43,47}$ S. H. Zhu, ${ }^{54}$ W. J. Zhu, ${ }^{34}$ X. L. Zhu, ${ }^{45}$ Y. C. Zhu, ${ }^{55,43}$ Y. S. Zhu, ${ }^{1,47}$ Z. A. Zhu, ${ }^{1,47}$ J. Zhuang, ${ }^{1,43}$ B.S. Zou, ${ }^{1}$ and J. H. Zou ${ }^{1}$

\section{(BESIII Collaboration)}

${ }^{1}$ Institute of High Energy Physics, Beijing 100049, People's Republic of China

${ }^{2}$ Beihang University, Beijing 100191, People's Republic of China

${ }^{3}$ Beijing Institute of Petrochemical Technology, Beijing 102617, People's Republic of China

${ }^{4}$ Bochum Ruhr-University, D-44780 Bochum, Germany

${ }^{5}$ Carnegie Mellon University, Pittsburgh, Pennsylvania 15213, USA

${ }^{6}$ Central China Normal University, Wuhan 430079, People's Republic of China

${ }^{7}$ China Center of Advanced Science and Technology, Beijing 100190, People's Republic of China

${ }^{8}$ COMSATS University Islamabad, Lahore Campus, Defence Road, Off Raiwind Road, 54000 Lahore, Pakistan

${ }^{9}$ Fudan University, Shanghai 200443, People's Republic of China

${ }^{10}$ G.I. Budker Institute of Nuclear Physics SB RAS (BINP), Novosibirsk 630090, Russia

${ }^{11}$ GSI Helmholtz centre for Heavy Ion Research GmbH, D-64291 Darmstadt, Germany

${ }^{12}$ Guangxi Normal University, Guilin 541004, People's Republic of China

${ }^{13}$ Guangxi University, Nanning 530004, People's Republic of China

${ }^{14}$ Hangzhou Normal University, Hangzhou 310036, People's Republic of China

${ }^{15}$ Helmholtz Institute Mainz, Johann-Joachim-Becher-Weg 45, D-55099 Mainz, Germany

${ }^{16}$ Henan Normal University, Xinxiang 453007, People's Republic of China

${ }^{17}$ Henan University of Science and Technology, Luoyang 471003, People's Republic of China

${ }^{18}$ Huangshan College, Huangshan 245000, People's Republic of China

${ }^{19}$ Hunan Normal University, Changsha 410081, People's Republic of China

${ }^{20}$ Hunan University, Changsha 410082, People's Republic of China

${ }^{21}$ Indian Institute of Technology Madras, Chennai 600036, India

${ }^{22}$ Indiana University, Bloomington, Indiana 47405, USA

${ }^{23 a}$ INFN Laboratori Nazionali di Frascati, I-00044 Frascati, Italy

${ }^{23 \mathrm{~b}}$ INFN and University of Perugia, I-06100 Perugia, Italy

${ }^{24 a}$ INFN Sezione di Ferrara, I-44122 Ferrara, Italy

${ }^{24 \mathrm{~b}}$ University of Ferrara, I-44122 Ferrara, Italy

${ }^{25}$ Institute of Physics and Technology, Peace Ave. 54B, Ulaanbaatar 13330, Mongolia

${ }^{26}$ Johannes Gutenberg University of Mainz, Johann-Joachim-Becher-Weg 45, D-55099 Mainz, Germany

${ }^{27}$ Joint Institute for Nuclear Research, 141980 Dubna, Moscow region, Russia

${ }^{28}$ Justus-Liebig-Universitaet Giessen, II. Physikalisches Institut, Heinrich-Buff-Ring 16,

D-35392 Giessen, Germany

${ }^{29}$ KVI-CART, University of Groningen, NL-9747 AA Groningen, Netherlands

${ }^{30}$ Lanzhou University, Lanzhou 730000, People's Republic of China

${ }^{31}$ Liaoning University, Shenyang 110036, People's Republic of China

${ }^{32}$ Nanjing Normal University, Nanjing 210023, People's Republic of China

${ }^{33}$ Nanjing University, Nanjing 210093, People's Republic of China

${ }^{34}$ Nankai University, Tianjin 300071, People's Republic of China

${ }^{35}$ Peking University, Beijing 100871, People's Republic of China

${ }^{36}$ Shandong Normal University, Jinan 250014, People's Republic of China

${ }^{37}$ Shandong University, Jinan 250100, People's Republic of China

${ }^{38}$ Shanghai Jiao Tong University, Shanghai 200240, People's Republic of China

${ }^{39}$ Shanxi University, Taiyuan 030006, People's Republic of China

${ }^{40}$ Sichuan University, Chengdu 610064, People's Republic of China

${ }^{41}$ Soochow University, Suzhou 215006, People's Republic of China

${ }^{42}$ Southeast University, Nanjing 211100, People's Republic of China

${ }^{43}$ State Key Laboratory of Particle Detection and Electronics, Beijing 100049, Hefei 230026, People's Republic of China

${ }^{44}$ Sun Yat-Sen University, Guangzhou 510275, People's Republic of China

${ }^{45}$ Tsinghua University, Beijing 100084, People's Republic of China ${ }^{46 a}$ Ankara University, 06100 Tandogan, Ankara, Turkey 


\author{
${ }^{46 \mathrm{~b}}$ Istanbul Bilgi University, 34060 Eyup, Istanbul, Turkey \\ ${ }^{46 c}$ Uludag University, 16059 Bursa, Turkey \\ ${ }^{46 \mathrm{~d}}$ Near East University, Nicosia, North Cyprus, Mersin 10, Turkey \\ ${ }^{47}$ University of Chinese Academy of Sciences, Beijing 100049, People's Republic of China \\ ${ }^{48}$ University of Hawaii, Honolulu, Hawaii 96822, USA \\ ${ }^{49}$ University of Jinan, Jinan 250022, People's Republic of China \\ ${ }^{50}$ University of Manchester, Oxford Road, Manchester M13 9PL, United Kingdom \\ ${ }^{51}$ University of Minnesota, Minneapolis, Minnesota 55455, USA \\ ${ }^{52}$ University of Muenster, Wilhelm-Klemm-Str. 9, 48149 Muenster, Germany \\ ${ }^{53}$ University of Oxford, Keble Rd, Oxford OX13RH, United Kingdom \\ ${ }^{54}$ University of Science and Technology Liaoning, Anshan 114051, People's Republic of China \\ ${ }^{55}$ University of Science and Technology of China, Hefei 230026, People's Republic of China \\ ${ }^{56}$ University of South China, Hengyang 421001, People's Republic of China \\ ${ }^{57}$ University of the Punjab, Lahore-54590, Pakistan \\ ${ }^{58 a}$ University of Turin, I-10125 Turin, Italy \\ ${ }^{58 \mathrm{~b}}$ University of Eastern Piedmont, I-15121 Alessandria, Italy \\ ${ }^{58 \mathrm{c}}$ INFN, I-10125 Turin, Italy \\ ${ }^{59}$ Uppsala University, Box 516, SE-75120 Uppsala, Sweden \\ ${ }^{60}$ Wuhan University, Wuhan 430072, People's Republic of China \\ ${ }^{61}$ Xinyang Normal University, Xinyang 464000, People's Republic of China \\ ${ }^{62}$ Zhejiang University, Hangzhou 310027, People's Republic of China \\ ${ }^{63}$ Zhengzhou University, Zhengzhou 450001, People's Republic of China
}

(Received 26 June 2019; published 30 September 2019; corrected 3 May 2022)

Using a data sample of $1.31 \times 10^{9} \mathrm{~J} / \psi$ events collected with the BESIII detector, a search for $\eta^{\prime} \rightarrow \gamma \gamma \eta$ via $J / \psi \rightarrow \gamma \eta^{\prime}$ is performed for the first time. No significant $\eta^{\prime}$ signal is observed in the $\gamma \gamma \eta$ invariant mass spectrum, and the branching fraction of $\eta^{\prime} \rightarrow \gamma \gamma \eta$ is determined to be less than $1.33 \times 10^{-4}$ at the $90 \%$ confidence level.

DOI: 10.1103/PhysRevD.100.052015

${ }^{\mathrm{a}}$ Also at Bogazici University, 34342 Istanbul, Turkey.
${ }^{\mathrm{b}}$ Also at the Moscow Institute of Physics and Techno Moscow 141700, Russia.

${ }^{\mathrm{c}}$ Also at the Functional Electronics Laboratory, Tomsk State University, Tomsk, 634050, Russia.

${ }^{\mathrm{d}}$ Also at the Novosibirsk State University, Novosibirsk, 630090, Russia.

"Also at the NRC "Kurchatov Institute", PNPI, 188300, Gatchina, Russia.

${ }^{\mathrm{f}}$ Also at Istanbul Arel University, 34295 Istanbul, Turkey.

${ }^{\mathrm{g}}$ Also at Goethe University Frankfurt, 60323 Frankfurt am Main, Germany.

${ }^{\mathrm{h}}$ Also at Key Laboratory for Particle Physics, Astrophysics and Cosmology, Ministry of Education; Shanghai Key Laboratory for Particle Physics and Cosmology; Institute of Nuclear and Particle Physics, Shanghai 200240, People's Republic of China.

${ }^{1}$ Also at Government College Women University, Sialkot51310. Punjab, Pakistan.

${ }^{\mathrm{j}}$ Also at Key Laboratory of Nuclear Physics and Ion-beam Application (MOE) and Institute of Modern Physics, Fudan University, Shanghai 200443, People's Republic of China.

${ }^{\mathrm{k}}$ Also at Harvard University, Department of Physics, Cambridge, Massachusetts 02138, USA.

Published by the American Physical Society under the terms of the Creative Commons Attribution 4.0 International license. Further distribution of this work must maintain attribution to the author(s) and the published article's title, journal citation, and DOI. Funded by SCOAP ${ }^{3}$.

\section{INTRODUCTION}

The $\eta^{\prime}$ meson provides a unique opportunity for understanding the distinct symmetry-breaking mechanisms present in low-energy quantum chromodynamics (QCD) [1-5], and its decays play an important role in exploring the effective theory of QCD at low energy [6]. Within the frameworks of the linear $\sigma$ model and the vector meson dominance (VMD) model [7,8], the branching fractions of $\eta^{\prime} \rightarrow \gamma \gamma \pi^{0}$ and $\eta^{\prime} \rightarrow \gamma \gamma \eta$ are predicted to be $3.8 \times 10^{-3}$ and $2.0 \times 10^{-4}$ [8], respectively. The dominant contributions come from the vector meson exchange processes, where for $\eta^{\prime} \rightarrow \gamma \gamma \pi^{0}$, the $\omega$ contributes $80.2 \%$ of the total VMD signal, while the $\rho$ contributes $4.6 \%$. For $\eta^{\prime} \rightarrow \gamma \gamma \eta, \rho$ and $\omega$ contribute $59.9 \%$ and $15.8 \%$, respectively.

Recently using $1.31 \times 10^{9} \mathrm{~J} / \psi$ events, BESIII reported the study of $\eta^{\prime} \rightarrow \gamma \gamma \pi^{0}$ for the first time, and the branching fraction of $\eta^{\prime} \rightarrow \gamma \gamma \pi^{0}$ was determined to be $(32.0 \pm 0.7 \pm$ $2.3) \times 10^{-4}$ [9]. By excluding the intermediate contributions from $\omega\left(\rho^{0}\right) \rightarrow \gamma \pi^{0}$, the so-called nonresonant branching fraction of $\eta^{\prime} \rightarrow \gamma \gamma \pi^{0}$ was determined to be $(6.16 \pm 0.64 \pm 0.67) \times 10^{-4}$ [9], which confirmed the theoretical prediction and indicated that this decay was dominated by the VMD processes. 
Unlike $\eta^{\prime} \rightarrow \gamma \gamma \pi^{0}$ decay, the $\eta^{\prime} \rightarrow \gamma \gamma \eta$ decay has not been observed to date. The most stringent upper limit, reported by GAMS- $4 \pi$ setup, on the branching fraction of this decay is $8 \times 10^{-4}$ at the $90 \%$ confidence level (CL) [10]. The BESIII experiment using $J / \psi$ radiative decays has observed a series of $\eta^{\prime}$ new decay modes [11-17], and in this paper we present a search for $\eta^{\prime} \rightarrow \gamma \gamma \eta$ in the $J / \psi$ radiative decay.

\section{DETECTOR AND MONTE CARLO SIMULATION}

The BESIII detector is a magnetic spectrometer [18] located at the Beijing Electron Position Collider (BEPCII) [19]. The cylindrical core of the BESIII detector consists of a helium-based multilayer drift chamber (MDC), a plastic scintillator time-of-flight system (TOF), and a CsI(Tl) electromagnetic calorimeter (EMC), which are all enclosed in a superconducting solenoidal magnet providing a $1.0 \mathrm{~T}$ (0.9 $\mathrm{T}$ in 2012) magnetic field. The solenoid is supported by an octagonal flux-return yoke with resistive plate counter muon identifier modules interleaved with steel. The acceptance of charged particles and photons is $93 \%$ over $4 \pi$ solid angle. The charged-particle momentum resolution at $1 \mathrm{GeV} / c$ is $0.5 \%$, and the $d E / d x$ resolution is $6 \%$ for the electrons from Bhabha scattering. The EMC measures photon energies with a resolution of $2.5 \%(5 \%)$ at $1 \mathrm{GeV}$ in the barrel (end cap) region. The time resolution of the TOF barrel part is $68 \mathrm{ps}$, while that of the end cap part is 110 ps.

Monte Carlo (MC) simulations are used to estimate backgrounds and determine the detection efficiencies. The GEANT4-based [20] simulation software BOOST [21] includes the geometric and material description of the BESIII detector, detector response, and digitization models, as well as the tracking of the detector running conditions and performance. Production of the charmonium state $J / \psi$ is simulated with KKMC [22], while the decays are generated with EVTGEN [23] for known decay modes with branching fractions taken from the Particle Data Group (PDG) [24] and by LUNDCHARM [25] for the remaining unknown decays.

In this analysis, the program EVTGEN is used to generate a $J / \psi \rightarrow \gamma \eta^{\prime}$ MC sample with an angular distribution of $1+\cos ^{2} \theta_{\gamma}$, where $\theta_{\gamma}$ is the polar angle of the radiative photon in the $J / \psi$ rest frame. The decays $\eta^{\prime} \rightarrow \gamma \omega(\rho)$, $\omega(\rho) \rightarrow \gamma \eta$ are generated using the VMD model $[7,8]$ with $\omega(\rho)$ exchange. For the nonresonant $\eta^{\prime} \rightarrow \gamma \gamma \eta$ decay, the VMD model is also used to generate the MC sample with $\omega(1420)$ or $\rho(1450)$ exchange. We use a sample of $1.225 \times$ $10^{9}$ simulated $J / \psi$ events to study the backgrounds in which the $J / \psi$ decays generically (inclusive MC sample). The analysis is performed in the framework of the BESIII offline software system [26] which incorporates the detector calibration, event reconstruction, and data storage.

\section{EVENT SELECTION AND BACKGROUND ESTIMATION}

In the reconstruction of $J / \psi \rightarrow \gamma \eta^{\prime}$ with $\eta^{\prime} \rightarrow \gamma \gamma \eta$ and $\eta \rightarrow \gamma \gamma$, candidate events must have no charged particle and at least five photons. Charged particles are identified by tracks in the active region of the MDC, corresponding to $|\cos \theta|<0.93$, where $\theta$ is the polar angle of the charged track with respect to the beam direction. They are also required to pass within $\pm 10 \mathrm{~cm}$ of the interaction point in the beam direction and $1 \mathrm{~cm}$ of the beam line in the plane perpendicular to the beam. The photon candidate showers must have minimum energy of $25 \mathrm{MeV}$ in the barrel region $(|\cos \theta|<0.8)$ or $50 \mathrm{MeV}$ in the end cap region $(0.86<|\cos \theta|<0.92)$. Showers in the region between the barrel and the end caps are poorly measured and excluded. A requirement of EMC cluster timing with respect to the most energetic photon $(-500 \mathrm{~ns}<\mathrm{T}<$ $500 \mathrm{~ns}$ ) is used to suppress electronic noise and energy deposits unrelated to the event. To select $J / \psi \rightarrow \gamma \eta^{\prime}, \eta^{\prime} \rightarrow$ $\gamma \gamma \eta(\eta \rightarrow \gamma \gamma)$ signal events, only the events with exactly five photon candidates are selected, and the most energetic photon is taken as the radiative photon from the $J / \psi$ decay.

A four-constraint (4C) kinematic fit imposing energymomentum conservation is performed to the $\gamma \gamma \gamma \gamma \gamma$ hypothesis and the $\chi^{2}$ is required to be less than 200. To distinguish the photons from $\eta^{\prime}$ and $\eta$ decays, a variable $\delta_{\eta^{\prime} \eta}^{2}$, defined as $\delta_{\eta^{\prime} \eta}^{2}=\left(\frac{M(\gamma \gamma \eta)-m\left(\eta^{\prime}\right)}{\sigma_{1}}\right)^{2}+\left(\frac{M(\gamma \gamma)-m(\eta)}{\sigma_{2}}\right)^{2}$, is introduced, where $M(\gamma \gamma \eta)$ is the invariant mass of four of five selected photons (expect for the radiative photon) for reconstructing the $\eta^{\prime}$ meson, $M(\gamma \gamma)$ is the invariant mass of photon pairs for reconstructing the $\eta$ meson, while $\sigma_{1}=$ $11.7 \mathrm{MeV} / c^{2}$ and $\sigma_{2}=9.7 \mathrm{MeV} / c^{2}$ are the mass resolutions of $\eta^{\prime}$ and $\eta$, respectively, obtained from the MC simulations, $m\left(\eta^{\prime}\right)$ and $m(\eta)$ are the $\eta^{\prime}$ and $\eta$ nominal masses, respectively. We then require $|M(\gamma \gamma)-m(\eta)|<$ $50 \mathrm{MeV} / c^{2}$ and the combination with the minimum value of $\delta_{\eta^{\prime} \eta}^{2}$ is chosen. Next the $\delta_{\eta^{\prime} \eta}^{2}$ is required to be less than $\delta_{\eta \eta}^{2}$, which is defined as $\delta_{\eta \eta}^{2}=\left(\frac{M_{1}(\gamma \gamma)-m(\eta)}{\sigma_{2}}\right)^{2}+\left(\frac{M_{2}(\gamma \gamma)-m(\eta)}{\sigma_{2}}\right)^{2}$, where $M_{1}(\gamma \gamma)$ and $M_{2}(\gamma \gamma)$ are the invariant masses of arbitrary two of five selected photons, to suppress the background events from $J / \psi \rightarrow \gamma \eta \eta$.

To improve the mass resolution and further suppress background events, a five-constraint (5C) kinematic fit imposing energy-momentum conservation with a $\eta$ mass constraint is performed under the $\gamma \gamma \gamma \eta$ hypothesis, where the $\eta$ candidate is reconstructed with the pair of photons described above, and the $\chi_{5 C}^{2}$ is required to be less than 30 . The $\chi_{5 C}^{2}$ distribution is shown in Fig. 1. In addition, the invariant masses of all the two photon pairs are required not to be in the $\pi^{0}$ mass region, $\left|M(\gamma \gamma)-m\left(\pi^{0}\right)\right|>$ $18 \mathrm{MeV} / c^{2}$, to suppress the background events with $\pi^{0}$ in the final state.

To remove the miscombinationed photon pairs in $\eta$ candidates, the $\eta$ decay angle $\theta_{\text {decay }}$, defined as the polar 


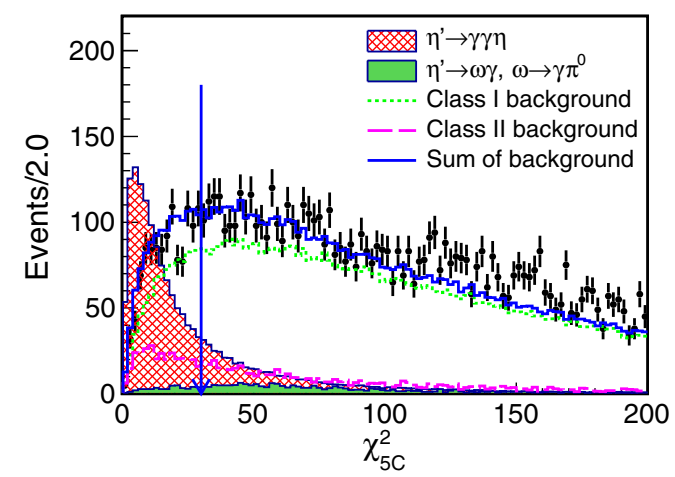

FIG. 1. $\chi_{5 C}^{2}$ distributions in MC simulations and data. Dots with error bars are data, the wide (blue) solid-curve is the sum of expected backgrounds from MC simulations, the grid area is from signal MC with arbitrary normalization, the (green) dotted-curve is the Class I $\left(J / \psi \rightarrow \gamma \eta^{\prime}, \eta^{\prime} \rightarrow \pi^{0} \pi^{0} \eta\right)$ background, the (pink) dashed-curve is the Class II $\left(J / \psi \rightarrow \gamma \eta \pi^{0}\right.$ and $J / \psi \rightarrow \omega \eta$ $\left(\omega \rightarrow \gamma \pi^{0}, \eta \rightarrow \gamma \gamma\right)$ ) background, and solid area is the peaking background.

angle of each photon in the corresponding $\gamma \gamma$ rest frame with respect to the $\eta$ direction in the $J / \psi$ rest frame, is required to satisfy $\left|\cos \theta_{\text {decay }}\right|<0.95$. An event is vetoed if any two of five selected photons (except for the combination for the $\eta$ candidate) satisfy $|M(\gamma \gamma)-m(\eta)|<35 \mathrm{MeV} / c^{2}$.
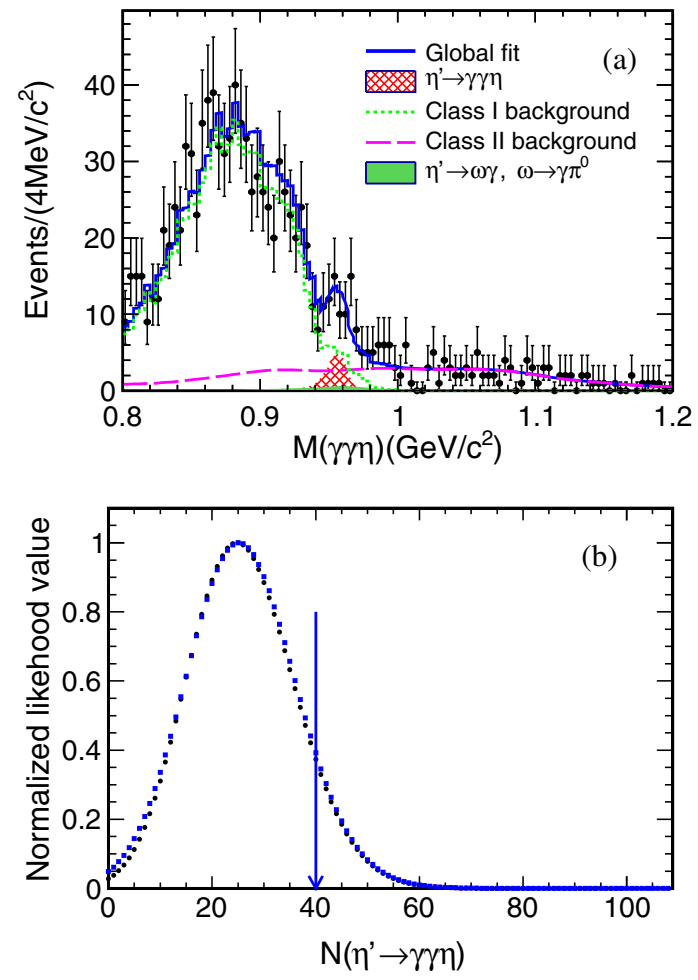

FIG. 2. (a) Results of the fit to $M(\gamma \gamma \eta)$. The black dots with error bars are data, and the others are the results of the fit described in the text. (b) Likelihood distribution before (black dots) and after (blue squares) taking into account systematic uncertainties [see Eq. (2)]. The arrow is the position of the upper limit on the signal yields at $90 \%$ CL.
The resulting $\gamma \gamma \eta$ invariant mass distribution, after these requirements, is shown in Fig. 2(a), where no significant $\eta^{\prime}$ peak is observed. Detailed MC studies indicate that the background events accumulating near the lower side of the $\eta^{\prime}$ signal region are mainly from $J / \psi \rightarrow \gamma \eta^{\prime}, \eta^{\prime} \rightarrow \pi^{0} \pi^{0} \eta$ (Class I), which is shown as the dotted (green) curve in Fig. 2(a). The peaking background is from $J / \psi \rightarrow \gamma \eta^{\prime}$, $\eta^{\prime} \rightarrow \gamma \omega, \omega \rightarrow \gamma \pi^{0}$, and is shown as the solid area in Fig. 2(a). The remaining background events are dominated by those $J / \psi$ decays without $\eta^{\prime}$ in the final states (Class II), e.g., $J / \psi \rightarrow \gamma \eta \pi^{0}$ and $J / \psi \rightarrow \omega \eta \quad\left(\omega \rightarrow \gamma \pi^{0}, \quad \eta \rightarrow \gamma \gamma\right)$ decays. They constitute a smooth distribution in the $\eta^{\prime}$ signal region as illustrated by the dashed (pink) curve in Fig. 2(a).

\section{SIGNAL YIELD AND BRANCHING FRACTION}

An unbinned maximum likelihood fit to the $M(\gamma \gamma \eta)$ distribution is performed to determine the $\eta^{\prime} \rightarrow \gamma \gamma \eta$ signal yield. In the fit, the probability density function (PDF) for the signal component is represented by the signal $\mathrm{MC}$ shape, which is obtained from the signal MC sample generated with an incoherent mixture of $\rho, \omega$ and the nonresonant components according to the fractions from the theoretical prediction $[7,8]$. The Class I and Class II background shapes are obtained from MC simulations and fixed, but the numbers are free parameters. Both the shape and the yield for the peaking background are fixed to the MC simulation and their expected intensities. The fit shown in Fig. 2(a) yields $24.9 \pm 10.3 \eta^{\prime} \rightarrow \gamma \gamma \eta$ events with a statistical significance of $2.6 \sigma$, and the branching fraction is calculated from

$B\left(\eta^{\prime} \rightarrow \gamma \gamma \eta\right)=\frac{N_{\mathrm{obs}}}{N_{J / \psi} \cdot \varepsilon \cdot B(\eta \rightarrow \gamma \gamma) \cdot B\left(J / \psi \rightarrow \gamma \eta^{\prime}\right)}$,

where $N_{\text {obs }}$ is the number of observed events determined from the fit to the $\gamma \gamma \eta$ mass spectrum, $\varepsilon$ is the MCdetermined detection efficiency, which is obtained from the signal MC sample described above; $B(\eta \rightarrow \gamma \gamma)$ and $B\left(J / \psi \rightarrow \gamma \eta^{\prime}\right)$ are the branching fractions of $\eta \rightarrow \gamma \gamma$ and $J / \psi \rightarrow \gamma \eta^{\prime}$ quoted from the PDG [24], respectively.

With the number of signal events and a detection efficiency of $11.4 \%$ the branching fraction is measured to be

$$
B\left(\eta^{\prime} \rightarrow \gamma \gamma \eta\right)=(8.25 \pm 3.41 \pm 0.72) \times 10^{-5},
$$

where the first uncertainty is statistical and the second systematic.

\section{SYSTEMATIC UNCERTAINTIES}

The systematic uncertainties on the upper limit measurement are summarized in Table I. The uncertainty due to the photon reconstruction is determined to be $0.5 \%$ per 
photon in the EMC barrel and $1.5 \%$ per photon in the EMC endcap [27]. Thus the uncertainty associated with the five reconstructed photons is $3 \%$ ( $0.6 \%$ per photon) by weighting the uncertainties according to the polar angle distribution of the five photons from data. The uncertainties associated with the other selection criteria, e.g., kinematic fit with $\chi_{5 C}^{2}<30$, the number of photons equal to $5, \pi^{0}$ veto $\left(\left|M(\gamma \gamma)-m\left(\pi^{0}\right)\right|>18 \mathrm{MeV} / c^{2}\right)$ and $\cos \theta_{\text {decay }}$, are studied using the $J / \psi \rightarrow \gamma \eta^{\prime} \rightarrow \gamma \gamma \omega, \omega \rightarrow \gamma \pi^{0}$ decay control sample [9]. The systematic uncertainty for each of the applied selection criteria is numerically estimated from the difference of the number of events with and without the corresponding requirement. The resultant efficiency differences between data and MC simulations $(2.7 \%, 0.5 \%$, $1.9 \%$, and $0.3 \%$, respectively) are taken as the corresponding systematic uncertainties.

To suppress the multi- $\eta$ backgrounds and remove the miscombinations of photons, an event is vetoed if any two of five selected photons (except for the combination for the $\eta$ candidate) satisfy $|M(\gamma \gamma)-m(\eta)|<35 \mathrm{MeV} / c^{2}$. To estimate the systematic uncertainty, this requirement varied by $\pm 10 \mathrm{MeV} / c^{2}$, and the maximum change to the nominal result is taken as the systematic uncertainty.

The signal shape is obtained from the MC simulation in the nominal fit for the $\eta^{\prime}$ decay. The uncertainty due to the signal shape is considered by convolving a Gaussian function to account for the difference in the mass resolution between data and MC simulation. In the fit to the $\gamma \gamma \eta$ distribution, the signal PDF is the signal MC shape convolving a Gaussian function with a fixed width of $1.5 \mathrm{MeV}$ [9], and the changes of the signal yields is taken as the uncertainty due to the signal shape.

The uncertainty due to the Class I background and the peaking background are estimated by varying the numbers of expected background events by one standard deviation according to the uncertainties on the branching fractions values in PDG [24].

TABLE I. Summary of relative systematic uncertainties for the upper limit on the branching fraction measurement (in \%).

\begin{tabular}{lc}
\hline \hline Source & Systematic uncertainties \\
\hline Photon detection & 3.0 \\
Kinematic fit $(5 \mathrm{C})$ & 2.7 \\
Number of photons $\left(N_{\gamma}=5\right)$ & 0.5 \\
$\pi^{0}$ and $\gamma$ veto & 1.9 \\
$\cos \theta_{\text {decay }}$ & 0.3 \\
$\eta$ veto & 4.3 \\
Signal shape & 3.2 \\
Class I background & 3.1 \\
Peaking background & 0.8 \\
Signal model & 2.9 \\
Cited branching fractions & 3.3 \\
Number of $J / \psi$ events & 0.5 \\
Total & 8.8 \\
\hline \hline
\end{tabular}

To take into account the systematic uncertainty due to signal model (VMD model), fits with alternative signal models for the different components, for example, a coherent sum for the $\rho, \omega$-components and a uniform angular distribution in phase space for the nonresonant process is performed. The resultant changes in the branching fractions (involving efficiency changes) are taken as the uncertainty related to the signal model.

To take into account the systematic uncertainties associated with the fit of the mass spectrum coming from the background events and the fit range, alternative fits with different fit ranges, background shapes and the number of background events are performed. The largest number of the signal yield among these cases is chosen to calculate the upper limit of the branching fraction at the 90\% CL.

The number of $J / \psi$ events is $N_{J / \psi}=(1310.6 \pm 7.0) \times$ $10^{6}$ [28], corresponding to an uncertainty of $0.5 \%$. The branching fractions for the $J / \psi \rightarrow \gamma \eta^{\prime}$ and $\eta \rightarrow \gamma \gamma$ decays are taken from the PDG [24], and the corresponding uncertainties are taken as a systematic uncertainty.

Assuming all systematic uncertainties in Table I are independent, the total systematic uncertainty, obtained from their quadratic sum, is $8.7 \%$.

\section{VI. $\boldsymbol{\eta}^{\prime} \rightarrow \gamma \gamma \boldsymbol{\eta}$ UPPER LIMIT RESULTS}

Since no significant $\eta^{\prime}$ peak is seen, we use the Bayesian method to obtain the signal upper limit. Unbinned maximum likelihood fits are performed on the $\gamma \gamma \eta$ mass spectrum with a series of input signal events, and the distribution of normalized likelihood values is taken as the PDF for the expected number of events.

The final upper limit on the branching fraction is determined by convolving the normalized likelihood curve $L(N)$ with the systematic uncertainties as a Gaussian function $\left[G(\mu, \sigma)=G\left(0, \sigma_{\text {sys }}\right)\right]$ to obtain the smeared likelihood $L^{\prime}\left(N^{\prime}\right)$, which is written as

$L^{\prime}\left(N^{\prime}\right)=\int_{0}^{\infty} L(N) \frac{1}{\sqrt{2 \pi} \sigma_{\mathrm{sys}}} \exp \left[\frac{-\left(N^{\prime}-N\right)^{2}}{2 \sigma_{\mathrm{sys}}^{2}}\right] d N$,

where $\sigma_{\text {sys }}=N \cdot \sigma_{\text {rel }}, N$ and $\sigma_{\text {rel }}$ are the input signal yield and the corresponding uncertainty, respectively. Figure 2(b) shows the likelihood distribution before and after convolving the Gaussian function. The upper limit on the number of $\eta^{\prime} \rightarrow \gamma \gamma \eta$ events, $N_{U L}^{\prime}$, is determined to be 40 at the $90 \% \mathrm{CL}$. The corresponding upper limit on the branching fraction of $\eta^{\prime} \rightarrow \gamma \gamma \eta$ is determined to be $B\left(\eta^{\prime} \rightarrow \gamma \gamma \eta\right)<$ $1.33 \times 10^{-4}$ at the $90 \%$ CL.

\section{SUMMARY}

With a data sample of $1.31 \times 10^{9} \mathrm{~J} / \psi$ events collected with the BESIII detector, we report on a search for the doubly radiative decay $\eta^{\prime} \rightarrow \gamma \gamma \eta$ for the first time, where the $\eta^{\prime}$ meson is produced via the $J / \psi \rightarrow \gamma \eta^{\prime}$ process. 
The observed signal yields in the $\gamma \gamma \eta$ invariant mass spectrum corresponds to $2.6 \sigma$, this signal corresponds to a branching fraction of $(8.25 \pm 3.41 \pm 0.72) \times 10^{-5}$. We also present an upper limit of the branching fraction of $1.33 \times 10^{-4}$ at the $90 \% \mathrm{CL}$. The obtained result is in tension with a recent theoretical prediction of $2.0 \times 10^{-4}$ [8] within the frame work of the linear $\sigma$ model and the VMD model.

\section{ACKNOWLEDGMENTS}

The BESIII collaboration thanks the staff of BEPCII and the IHEP computing center for their strong support. This work is supported in part by National Key Basic Research Program of China under Contract No. 2015CB856700; National Natural Science Foundation of China (NSFC) under Contracts No. 11625523, No. 11635010, No. 11675184, No. 11735014, No. 11835012; the Chinese Academy of Sciences (CAS) Large-Scale Scientific Facility Program; Joint Large-Scale Scientific Facility Funds of the NSFC and CAS under Contracts
No. U1532257, No. U1532258, No. U1732263, No. U1832207; CAS Key Research Program of Frontier Sciences under Contracts No. QYZDJ-SSW-SLH003, No. QYZDJ-SSW-SLH040; 100 Talents Program of CAS; INPAC and Shanghai Key Laboratory for Particle Physics and Cosmology; German Research Foundation DFG under Contracts No. Collaborative Research Center CRC 1044, FOR 2359; Istituto Nazionale di Fisica Nucleare, Italy; Koninklijke Nederlandse Akademie van Wetenschappen (KNAW) under Contract No. 5304CDP03; Ministry of Development of Turkey under Contract No. DPT2006K-120470; National Science and Technology fund; The Knut and Alice Wallenberg Foundation (Sweden) under Contract No. 2016.0157; The Royal Society, UK under Contract No. DH160214; The Swedish Research Council; U.S. Department of Energy under Contracts No. DE-FG02-05ER41374, No. DE-SC-0010118, No. DE-SC-0012069; University of Groningen $(\mathrm{RuG})$ and the Helmholtzzentrum fuer Schwerionenforschung GmbH (GSI), Darmstadt.
[1] J. Steinberger, Phys. Rev. 76, 1180 (1949); S. L. Adler, Phys. Rev. 177, 2426 (1969); J. S. Bell and R. Jackiw, Nuovo Cimento A 60, 47 (1969); W. A. Bardeen, Phys. Rev. 184, 1848 (1969).

[2] J. Wess and B. Zumino, Phys. Lett. 37B, 95 (1971).

[3] E. Witten, Nucl. Phys. B223, 422 (1983).

[4] H. B. Li, J. Phys. G 36, 085009 (2009).

[5] A. Kupsc, Int. J. Mod. Phys. E 18, 1255 (2009).

[6] J. Gasser and H. Leutwyler, Nucl. Phys. B250, 465 (1985); H. Neufeld and H. Rupertsberger, Z. Phys. C 68, 91 (1995).

[7] R. Jora, Nucl. Phys. B, Proc. Suppl. 207, 224 (2010).

[8] R. Escribano et al., arXiv:1812.08454.

[9] M. Ablikim et al. (BESIII Collaboration), Phys. Rev. D 96, 012005 (2017).

[10] S. V. Donskov, V. N. Kolosov, A. A. Lednev, Y. V. Mikhailov, V. A. Polyakov, V. D. Samoylenko, and G. V. Khaustov, Phys. At. Nucl. 78, 1043 (2015).

[11] M. Ablikim et al. (BESIII Collaboration), Phys. Rev. D 97, 012003 (2018).

[12] M. Ablikim et al. (BESIII Collaboration), Phys. Rev. Lett. 120, 242003 (2018).

[13] M. Ablikim et al. (BESIII Collaboration), Phys. Rev. D 92, 012014 (2015).

[14] M. Ablikim et al. (BESIII Collaboration), Phys. Rev. Lett. 118, 012001 (2017).

[15] M. Ablikim et al. (BESIII Collaboration), Phys. Rev. D 92, 012001 (2015).

[16] M. Ablikim et al. (BESIII Collaboration), Phys. Rev. D 87, 092011 (2013).
[17] M. Ablikim et al. (BESIII Collaboration), Phys. Rev. Lett. 112, 251801 (2014).

[18] M. Ablikim et al. (BESIII Collaboration), Nucl. Instrum. Methods Phys. Res., Sect. A 614, 345 (2010).

[19] C. H. Yu et al., Proceedings of IPAC2016, Busan, Korea (2016), https://dx.doi.org/10.18429/JACoW-IPAC2016TUYA01.

[20] S. Agostinelli et al. (GEANT4 Collaboration), Nucl. Instrum. Methods Phys. Res., Sect. A 506, 250 (2003).

[21] Z. Y. Deng et al., Chin. Phys. C 30, 371 (2006).

[22] S. Jadach, B.F. L. Ward, and Z. Was, Comput. Phys. Commun. 130, 260 (2000); Phys. Rev. D 63, 113009 (2001).

[23] D. J. Lange, Nucl. Instrum. Methods Phys. Res., Sect. A 462, 152 (2001); R. G. Ping, Chin. Phys. C 32, 599 (2008).

[24] M. Tanabashi et al. (Particle Data Group), Phys. Rev. D 98, 030001 (2018).

[25] J. C. Chen, G. S. Huang, X. R. Qi, D. H. Zhang, and Y. S. Zhu, Phys. Rev. D 62, 034003 (2000).

[26] W. D. Li et al., in Proceeding of CHEP06, Mumbai, India, 2006, edited by Sunanda Banerjee (Tata Institute of Fundamental Research, Mumbai, 2006).

[27] M. Ablikim et al. (BESIII Collaboration), Phys. Rev. D 96, 112012 (2017).

[28] M. Ablikim et al. (BESIII Collaboration), Chin. Phys. C 41, 013001 (2017).

Correction: A second affiliation indicator was missing for author Y. Q. Wang and has been added. 